\title{
LARGE REGULAR SIMPLICES CONTAINED IN A HYPERCUBE
}

\author{
HIROSHI MAEHARA, IMRE Z. RUZSA, AND NORIHIDE TOKUSHIGE
}

\begin{abstract}
We prove that the $n$-dimensional unit hypercube contains an $n$-dimensional regular simplex of edge length $c \sqrt{n}$, where $c>0$ is a constant independent of $n$.
\end{abstract}

Let $\ell \Delta_{n}$ be the $n$-dimensional regular simplex of edge length $\ell$, and let $\ell Q_{n}$ be the $n$-dimensional hypercube of edge length $\ell$. For simplicity, we omit $\ell$ if $\ell=1$, e.g., $Q_{n}$ denotes the unit hypercube. We are interested in the maximum edge length of a regular $n$-dimensional simplex contained in $Q_{n}$.

Theorem. For every $\varepsilon_{0}>0$ there is an $N_{0}$ such that for every $n>N_{0}$ one has

$$
\left(\frac{1-\varepsilon_{0}}{2} \sqrt{n}\right) \Delta_{n} \subset Q_{n}
$$

On the other hand, if $\ell \Delta_{n} \subset Q_{n}$, then $\ell \leq \sqrt{(n+1) / 2}$, which follows by comparing the circumscribed balls of $\ell \Delta_{n}$ and $Q_{n}$. (Recall that the circumradius of $\Delta_{n}$ is $\sqrt{n /(2 n+2)}$.) This upper bound is reached iff there exists an Hadamard matrix of order $n+1$. Schoenberg [3] pointed out that this "readily established fact" went back to Coxeter, see also $\S 4$ of [1]. Our lower bound given by the theorem is approximately $1 / \sqrt{2}$ of the upper bound.

Proof of Theorem. For a matrix (or a vector) $A=\left(a_{i j}\right)$, let us define its norm by $\|A\|:=\max _{i j}\left|a_{i j}\right|$. Let $J_{n}$ be the $n \times n$ all one matrix.

Lemma 1. Let $A=\left(a_{i j}\right)$ be an $n \times n$ real orthogonal matrix, and let $c>0$ be a constant. If

$$
\|A\| \leq \frac{1}{c \sqrt{n}}
$$

Date: November 18, 2008.

2000 Mathematics Subject Classification. Primary: 52C07 Secondary: 05B20.

Key words and phrases. regular simplex, hypercube, Hadamard matrix.

The first and the last authors were supported by MEXT Grant-in-Aid for Scientific Research (B) 20340022.

Second author supported by Hungarian National Research Fund (OTKA), grants No. K 61908, K 72731. 
and

$$
\left\|J_{n} A\right\| \leq \frac{1}{c}
$$

then we have $(c \sqrt{n / 2}) \Delta_{n} \subset Q_{n}$.

Proof. Let $p_{i}=\left(a_{i 1}, \ldots, a_{i n}\right)$ be the $i$-th row of the matrix $A$. Then the $n$ points $p_{1}, \ldots, p_{n} \in \mathbb{R}^{n}$ form a $\sqrt{2} \Delta_{n-1}$. By (1), we have $\left\|p_{i}\right\| \leq 1 /(c \sqrt{n})$ for all $1 \leq i \leq n$.

Let $g=\left(g_{1}, \ldots, g_{n}\right) \in \mathbb{R}^{n}$ be the barycenter of the above $\sqrt{2} \Delta_{n-1}$, and let $p_{n+1}:=(1-\sqrt{n+1}) g$. Then a computation shows that the $p+1$ points $p_{1}, \ldots, p_{n}, p_{n+1} \in \mathbb{R}^{n}$ form a $\sqrt{2} \Delta_{n}$. Moreover, it follows from (2) that $\left\|p_{n+1}\right\|=\|g\|(\sqrt{n+1}-1) \leq 1 /(c \sqrt{n})$. Thus we have $\sqrt{2} \Delta_{n} \subset(2 /(c \sqrt{n})) Q_{n}$, as desired.

Let us find orthogonal matrices satisfying (1) and (2). Let $q$ be an odd prime power, and let $\mathbb{F}_{q}=\left\{b_{0}, \ldots, b_{q-1}\right\}\left(b_{0}=0\right)$ be the finite field of order $q$. Define a character $\chi: \mathbb{F}_{q} \rightarrow\{0, \pm 1\}$ by $\chi(0)=0, \chi(x)=1$ if $x$ is a square, and $\chi(x)=-1$ if $x$ is a nonsquare. Define an $q \times q$ matrix $B=$ $\left(b_{i j}\right)$ by setting $b_{i j}:=\chi\left(b_{i}-b_{j}\right)$. Then this matrix satisfies $B B^{T}=q I_{q}-J_{q}$, $B J_{q}=J_{q} B=O$. (See pp. 202-203 in [2] for the proof and how to use this matrix to construct an Hadamard matrix of Paley type.) Finally we define an orthogonal $q \times q$ matrix $A_{q}$ by

$$
A_{q}:=\frac{1}{\sqrt{q}}\left(B+\frac{1}{\sqrt{q}} J_{q}\right)
$$

Then, it is easy to check that $A_{q}$ satisfies $\left\|A_{q}\right\| \leq \frac{1}{\sqrt{q}}+\frac{1}{q}$ and $J_{q} A_{q}=J_{q}$. Thus the matrix $A_{q}$ satisfies (1) and (2) for $c=1-o(1)$, and this verifies the theorem for the case when the dimension is an odd prime power. (By using the fact that each entry $a_{i j}$ of $A_{q}$ satisfies $\left|a_{i j}-1 / q\right| \leq 1 / \sqrt{q}$, instead of (1), we can remove the $o(1)$, i.e., we actually get $\sqrt{q / 2} \Delta_{q} \subset Q_{q}$.)

Now let $q_{1}, \ldots, q_{r}$ be distinct odd prime powers, and let $n=q_{1} \cdots q_{r}$ and $A_{n}:=A_{q_{1}} \otimes \cdots \otimes A_{q_{r}}$. Then the matrix $A_{n}$ is orthogonal with

$$
\left\|A_{n}\right\| \leq \frac{1}{\sqrt{n}} \prod_{i=1}^{r}\left(1+\frac{1}{\sqrt{q_{i}}}\right) .
$$

Moreover, $A_{n}$ satisfies $J_{n} A_{n}=J_{n}$ because $J_{n} A_{n}=\left(J_{q_{1}} \otimes \cdots \otimes J_{q_{r}}\right)\left(A_{q_{1}} \otimes \cdots \otimes\right.$ $\left.A_{q_{r}}\right)=\left(J_{q_{1}} A_{q_{1}}\right) \otimes \cdots \otimes\left(J_{q_{r}} A_{q_{r}}\right)=J_{n}$. We notice that

$$
\prod_{i=1}^{r}\left(1+\frac{1}{\sqrt{q_{i}}}\right) \leq \prod_{p \mid n}\left(1+\frac{1}{\sqrt{p}}\right)=\sum_{d \mid n} \frac{1}{\sqrt{d}}=: g(n),
$$


where the product in the middle term is taken for all primes $p$ dividing $n$. Thus (3) gives (1) with $c=1 / g(n)$, and Lemma 1 implies that

$$
\left(\frac{\sqrt{n}}{g(n) \sqrt{2}}\right) \Delta_{n} \subset Q_{n}
$$

for every odd integer $n$.

Lemma 2. For every $\varepsilon, \delta>0$ there is an $n_{0}$ with the following property. For every integer $n>n_{0}$ there are odd integers $n_{1}, n_{2}$ such that $2 n=n_{1}+n_{2}$, $(1-\varepsilon) n \leq n_{i} \leq(1+\varepsilon) n$ and $g\left(n_{i}\right)<1+\delta$ for each $i=1,2$.

Proof. We will select an $m$, define

$$
q=\prod_{p \leq m} p
$$

as the product of the primes up to $m$ and select $n_{1}, n_{2}$ coprime to $q$. This guarantees that they are odd.

First we average $g(n)$ over integers coprime to $q$. Let $(q, r)=1$. Write $I_{r}$ for the set of integers $\{j \in[(1-\varepsilon) n,(1+\varepsilon) n]: j \equiv r(\bmod q)\}$. We have

$$
\sum_{j \in I_{r}}(g(j)-1)=\sum_{d>1} \frac{1}{\sqrt{d}} N_{d},
$$

where $N_{d}$ is the number of multiples of $d$ in our set $I_{r}$. Clearly $N_{d}=0$ if $(d, q)>1$. If $(d, q)=1$, then the multiples of $d$ in this residue class form an arithmetic progression with difference $q d$, and we have the estimate

$$
N_{d} \leq \frac{2 \varepsilon n}{q d}+1 \text {. }
$$

Furthermore $N_{d}=0$ if $d \geq 2 n$.

Our choice of $q$ implies that whenever $d>1$ and $(d, q)=1$, then $d>m$, so we have

$$
\sum_{j \in I_{r}}(g(j)-1) \leq \sum_{m<d<2 n} \frac{1}{\sqrt{d}}\left(\frac{2 \varepsilon n}{q d}+1\right) .
$$

We use the easy estimates

$$
\sum_{d>m} d^{-3 / 2}<2 / \sqrt{m}, \sum_{d<2 n} d^{-1 / 2}<2 \sqrt{2 n}<3 \sqrt{n}
$$

to conclude

$$
\sum_{j \in I_{r}}(g(j)-1)<\frac{4 \varepsilon n}{q \sqrt{m}}+3 \sqrt{n} .
$$

Now we define $r$ as follows. Take a prime $p \leq m$. If $2 n \not \equiv 1(\bmod p)$, we put $r \equiv 1(\bmod p)$; if $2 n \equiv 1(\bmod p)$, let $r \equiv 2(\bmod p)$. In this way 
both $r$ and $r^{\prime}=2 n-r$ will be coprime to $q$. Applying (6) for $r$ and $r^{\prime}$ and summing we get

$$
\sum_{j \in I_{r}}((g(j)-1)+(g(2 n-j)-1))<\frac{8 \varepsilon n}{q \sqrt{m}}+6 \sqrt{n} .
$$

The number of summands in the above sum is $\geq 2 \varepsilon n / q-1>\varepsilon n / q$, assuming that $q<\varepsilon n$. Hence there is a value $j$ such that

$$
(g(j)-1)+(g(2 n-j)-1)<\frac{8}{\sqrt{m}}+\frac{6 q}{\varepsilon \sqrt{n}} .
$$

If the right hand side is $<\delta$, we are done. To achieve this we make both summands $<\delta / 2$. First we choose $m$ so that $8 / \sqrt{m}<\delta / 2$, that is, $m>$ $(16 / \delta)^{2}$. This determines the value of $q$, and we choose $n$ so large that $6 q /(\varepsilon \sqrt{n})<\delta / 2$, that is, $n>(12 q /(\varepsilon \delta))^{2}$.

Lemma 3. Let $\ell>0$ be a real, and let $s$ and $t$ be positive integers with $\ell^{2} \leq s \leq t$. If $\ell \Delta_{s} \subset Q_{s}$ and $\ell \Delta_{t} \subset Q_{t}$, then $\ell \Delta_{s+t+1} \subset Q_{s+t+1}$.

Proof. Let $p_{0}, p_{1}, \ldots, p_{s}$ be the vertices of $\ell \Delta_{s}$ inside $Q_{s}$, and let $q_{0}, q_{1}, \ldots, q_{t}$ be the vertices of $\ell \Delta_{t}$ inside $Q_{t}$. We may assume that the origin is the centers of these regular simplices. Then the distance between $p_{i}$ and the origin is given by $\ell \sqrt{s /(2 s+2)}$. We will construct $\ell \Delta_{s+t+1}$ with vertices $u_{0}, \ldots, u_{s}, v_{0}, \ldots, v_{t}$ as follows. Define $u_{i}$ for $0 \leq i \leq s$ and $v_{j}$ for $0 \leq j \leq t$ by

$$
u_{i}=\left(p_{i}, \overrightarrow{0}, x\right) \in \mathbb{R}^{s} \times \mathbb{R}^{t} \times \mathbb{R}, \quad v_{j}=\left(\overrightarrow{0}, q_{j}, 0\right) \in \mathbb{R}^{s} \times \mathbb{R}^{t} \times \mathbb{R} .
$$

Choose $x>0$ so that $\left|u_{i}-v_{j}\right|=\ell$ for all $i, j$. This can be done by solving

$$
\left|u_{i}-v_{j}\right|^{2}=\frac{s}{2 s+2} \ell^{2}+\frac{t}{2 t+2} \ell^{2}+x^{2}=\ell^{2},
$$

which gives $x=\ell\left(\frac{1}{2 s+2}+\frac{1}{2 t+2}\right)^{1 / 2}<\ell / \sqrt{s+1}<1$. Namely, we have

$$
u_{i}, v_{j} \in Q_{s} \times Q_{t} \times[0,1]=Q_{s+t+1},
$$

for all $i, j$.

We are ready to prove the theorem. Let $\varepsilon_{0}>0$ be given. Set $\varepsilon=\varepsilon_{0} / 2$ and take $\delta>0$ so that

$$
1-\varepsilon=\sqrt{1-\varepsilon} /(1+\delta) .
$$

Plug these $\varepsilon$ and $\delta$ into Lemma 2 to get $k_{0}=k_{0}(\varepsilon, \delta)>0$ such that for every $k>k_{0}$ there are $k_{1}, k_{2}$ satisfying $2 k=k_{1}+k_{2}, k_{i} \geq(1-\varepsilon) k$, and $g\left(k_{i}\right)<1+\delta$. Choose $N_{0} \geq 2 k_{0}$ so that

$$
(1-\varepsilon) \sqrt{n-1}>\left(1-\varepsilon_{0}\right) \sqrt{n}
$$

holds for all $n>N_{0}$. 
Now, let $n>N_{0}$ be given. First assume that $n$ is odd, and write $n=2 k+1$. Lemma 2 gives a decomposition $2 k=k_{1}+k_{2}$. Then we have $\ell_{i} \Delta_{k_{i}} \subset Q_{k_{i}}$ for $i=1,2$, where

$$
\ell_{i} \stackrel{(5)}{=} \frac{\sqrt{k_{i}}}{g\left(k_{i}\right) \sqrt{2}}>\frac{\sqrt{(1-\varepsilon) k}}{(1+\delta) \sqrt{2}} \stackrel{(7)}{=} \frac{(1-\varepsilon) \sqrt{k}}{\sqrt{2}}=\frac{1-\varepsilon}{2} \sqrt{n-1} \stackrel{(8)}{>} \frac{1-\varepsilon_{0}}{2} \sqrt{n} .
$$

Applying Lemma 3 with $s=k_{1}, t=k_{2}$ and $\ell=\frac{1-\varepsilon_{0}}{2} \sqrt{n}$, we have the desired result $\ell \Delta_{n} \subset Q_{n}$.

Next assume that $n$ is even, and write $n=2 k$. Lemma 2 gives $2 k=k_{1}+k_{2}$ and

$$
\left\|A_{k_{i}}\right\| \stackrel{(3)(4)}{\leq} \frac{g\left(k_{i}\right)}{\sqrt{k_{i}}}<\frac{1+\delta}{\sqrt{(1-\varepsilon) k}} \stackrel{(7)}{=} \frac{\sqrt{2}}{(1-\varepsilon) \sqrt{n}}<\frac{\sqrt{2}}{\left(1-\varepsilon_{0}\right) \sqrt{n}}=: \frac{1}{c \sqrt{n}} .
$$

Define an $n \times n$ orthogonal matrix $C$ by

$$
C=\left(\begin{array}{cc}
A_{k_{1}} & 0 \\
0 & A_{k_{2}}
\end{array}\right) \text {. }
$$

Then we have $\|C\| \leq \max \left\|A_{k_{i}}\right\|<1 /(c \sqrt{n})$ and $\left\|J_{n} C\right\|=\max \left\|J_{k_{i}} A_{k_{i}}\right\|=$ 1. Thus, by Lemma 1 , we have $(c \sqrt{n / 2}) \Delta_{n}=\left(\frac{1-\varepsilon_{0}}{2} \sqrt{n}\right) \Delta_{n} \subset Q_{n}$. This completes the proof of the theorem.

\section{REFERENCES}

[1] M. Hudelson, V. Klee, D. Larman. Largest $j$-simplices in $d$-cubes: some relatives of the Hadamard maximum determinant problem. Linear Algebra Appl. 241/243 (1996) 519-598.

[2] J. H. van Lint, R. M. Wilson. A course in combinatorics. Second edition. Cambridge University Press, Cambridge, 2001.

[3] I. J. Schoenberg. Regular simplices and quadratic forms. Journal of the London Mathematical Society 12 (1937) 48-55.

College of Education, Ryukyu University, Nishihara, Okinawa, 9030213 JAPAN

E-mail address: hmaehara@edu.u-ryukyu.ac.jp

Alfréd Rényi institute of Mathematics, Hungarian ACAdemy of SciENCES, BudAPEST, PF. 127, H-1364 HungARY

E-mail address: ruzsa@renyi . hu

College of Education, Ryukyu University, Nishihara, Okinawa, 903 0213 JAPAN

E-mail address: hide@edu.u-ryukyu.ac.jp 